\title{
Phase II Prospective, Open-Label Randomized Controlled Trial Comparing Standard of Care Chemotherapy With and Without Sequential Cytoreductive Interventions for Patients with Oligometastatic Foregut Adenocarcinoma and Undetectable Circulating Tumor Deoxyribose Nucleic Acid (ctDNA) Levels
}

\author{
Ankit Dhiman, MBBS, MS ${ }^{1}$, Charles C. Vining, MD ${ }^{1}$, Hunter D. D. Witmer, MD ${ }^{1}$, Divya Sood, MD ${ }^{1}$, \\ Ardaman Shergill, $\mathrm{MD}^{2}$, Hedy Kindler, $\mathrm{MD}^{2}$, Kevin K. Roggin, MD ${ }^{1}$, Mitchell C. Posner, MD ${ }^{1}$, \\ Osmanuddin S. Ahmed, $\mathrm{MD}^{3}$, Stanley Liauw, $\mathrm{MD}^{4,5}$, Sean Pitroda, $\mathrm{MD}^{4,5}$, Chih-Yi Liao, $\mathrm{MD}^{2}$, \\ Theodore Karrison, $\mathrm{PhD}^{6}$, Ralph Weichselbaum, $\mathrm{MD}^{4,5}$, Blase Polite, $\mathrm{MD}^{2}$, Oliver $\mathrm{S}$. Eng, $\mathrm{MD}^{1}$, \\ Daniel V. T. Catenacci, MD $^{2}$, and Kiran K. Turaga, MD, MPH ${ }^{1}$
}

\footnotetext{
${ }^{1}$ Section of General Surgery and Surgical Oncology, Department of Surgery, University of Chicago Medicine, Chicago, IL; ${ }^{2}$ Section of Hematology/Oncology, Department of Medicine, University of Chicago Medicine, Chicago, IL; ${ }^{3}$ Department of Radiology, University of Chicago, Chicago, IL; ${ }^{4}$ Department of Radiation and Cellular Oncology, University of Chicago, Chicago, IL; ${ }^{5}$ The Ludwig Center for Metastasis Research, University of Chicago, Chicago, IL; ${ }^{6}$ Department of Public Health Sciences, University of Chicago, Chicago, IL
}

\begin{abstract}
Background. Metastatic adenocarcinomas of foregut origin are aggressive and have limited treatment options, poor quality of life, and a dismal prognosis. A subset of such patients with limited metastatic disease might have favorable outcomes with locoregional metastasis-directed therapies. This study investigates the role of sequential cytoreductive interventions in addition to the standard of care chemotherapy in patients with oligometastatic foregut adenocarcinoma.

Methods. This is a single-center, phase II, open-label randomized clinical trial. Eligible patients include adults
\end{abstract}

Ankit Dhiman, Charles C. Vining, Daniel V. T. Catenacci and Kiran $\mathrm{K}$. Turaga have contributed equally to this work.

(C) Society of Surgical Oncology 2022

First Received: 4 August 2021

Accepted: 7 December 2021; Published Online: 5 January 2022

K. K. Turaga, MD, MPH

e-mail: Kturaga@gmail.com;

kturaga@surgery.bsd.uchicago.edu with synchronous or metachronous oligometastatic (metastasis limited to two sites and amenable for curative/ ablative treatment) adenocarcinoma of the foregut without progression after induction chemotherapy and having undetectable ctDNA. These patients will undergo induction chemotherapy and will then be randomized (1:1) to either sequential curative intervention followed by maintenance chemotherapy versus routine continued chemotherapy. The primary endpoint is progression-free survival (PFS), and a total of 48 patients will be enrolled to detect an improvement in the median PFS in the intervention arm with a hazard ratio (HR) of 0.5 with $80 \%$ power and a one-sided alpha of 0.1 . Secondary endpoints include disease-free survival (DFS) in the intervention arm, overall survival (OS), ctDNA conversion rate pre/post-induction chemotherapy, ctDNA PFS, $\mathrm{PFS}_{2}$, adverse events, quality of life, and financial toxicity.

Discussion. This is the first randomized study that aims to prospectively evaluate the efficacy and safety of surgical/ ablative interventions in patients with ctDNA-negative oligometastatic adenocarcinoma of foregut origin post-induction chemotherapy. The results from this study will likely develop pertinent, timely, and relevant knowledge in oncology. 
Metastatic adenocarcinomas of foregut origin (esophagus, gastric, duodenal, biliary, ampullary and pancreatic) are aggressive, ${ }^{1}$ and have limited treatment options, poor quality of life, and a dismal prognosis. ${ }^{2-5}$ A subset of patients with limited metastatic disease ${ }^{2-4}$ have favorable outcomes with locoregional therapies such as metastasectomy. Identifying this cohort of patients that might benefit from curative intent cytoreductive therapies in metastatic foregut adenocarcinomas is challenging. ${ }^{6-13}$

Oligometastasis is a distinct disease state between locally confined tumor and widespread systemic metastasis. ${ }^{14,15}$ It is based on the hypothesis that the evolution of metastatic disease has intermediary states in which disease is confined to a limited number of sites or organs. ${ }^{14-16}$ Therefore, local therapies including surgical resection of this limited metastatic disease may confer a survival benefit. Over the past decade, prospective randomized studies have confirmed the role of metastasis-directed interventions with improvement in oncological outcomes in many solid cancers such as non-small-cell lung cancer (NSCLC),${ }^{17}$ colorectal cancer, ${ }^{18,} 19$ and prostate cancer, ${ }^{20}$ with a subset of patients achieving long-term disease-free survival. Whether actively reducing tumor burden leads to improved PFS and, by extension, OS, or whether merely having a lower tumor burden at diagnosis is indicative of lead-time bias and/or favorable disease biology remains unknown. In addition to evaluating such active tumor reduction with an appropriate randomized control, carefully identifying such patients with oligometastatic disease who may benefit from locoregional surgical interventions may improve oncologic outcomes.

Circulating tumor deoxyribonucleic acid (ctDNA), or fragmented, tumor-derived DNA molecules circulating in the bloodstream is rapidly emerging as a non-invasive diagnostic and prognostic biomarker for several different malignancies. ${ }^{21-26}$ The fraction of patients with detectable ctDNA, as well as its concentration within the plasma, is known to correlate with increasing tumor stage. ${ }^{27}$ Recent systematic reviews have found that patients with lower levels of circulating stigmata of cancer tend to have better overall survival ${ }^{28}$ and that such markers are beneficial in identifying patients experiencing biochemical and clinical responses to systemic therapy. ${ }^{28,}{ }^{29}$ Traditional tumor markers such as CEA, CA 19-9, and AFP have varying specificity, limited prognostic value, and can be misleadingly elevated in benign conditions. In contrast, ctDNA is more specific, with levels of detection correlating with malignant progression. ${ }^{30}$ Moreover, ctDNA has been established as a valuable tool for predicting prognosis before and after therapy for different cancers ${ }^{31}$ and has demonstrated promise for the detection of recurrence of cancer following resection. ${ }^{32-34}$
To our knowledge, this randomized study is unique in evaluating the efficacy and safety of systematic selection of patients with oligometastatic disease, without detectable ctDNA after induction chemotherapy, for sequential cytoreductive interventions.

\section{METHODS}

This protocol summary follows the Standard Protocol Items: Recommendations for Interventional Trials (SPIRIT) Statement. ${ }^{35}$

\section{Design and Setting}

This study is a single-center, phase II, open-label randomized clinical trial assessing the role of sequential cytoreductive interventions in addition to standard of care chemotherapy compared with chemotherapy alone in patients with oligometastatic foregut adenocarcinoma.

\section{Eligibility}

A total of 48 consecutive patients presenting to the University of Chicago Medical Center with synchronous or metachronous oligometastatic adenocarcinoma of the foregut will be enrolled for this trial. Patients with esophageal, gastroesophageal junction, gastric, duodenal, or ampullary adenocarcinoma will be considered as having intestinal-type cancer, while those with cholangiocarcinoma or adenocarcinoma of the pancreas or gall bladder will be considered to be the pancreaticobiliary type. For patients with synchronous metastasis, the primary site must be either resectable or amenable to definitive interventions (radiation, chemoradiation, or ablation). Furthermore, for those presenting with metachronous metastasis, the distant disease must have developed at least 6 months after completion of previous curative-intent treatment of the primary site. The oligometastatic state in the study is defined as the presence of limited metastatic disease, limited to two sites amenable to either complete surgical resection and/or ablative interventions. Metastatic sites may include lung, liver, non-regional lymph nodes, or peritoneal disease. The number of metastatic lesions must be limited to five total combined for both lung and liver sites. The non-regional lymph nodes are defined according to the primary (Appendix A, Supplementary file), and must be limited to two sites. Similarly, the peritoneal lesions must have a combined Peritoneal Carcinomatosis Index (PCI) of less than six and should be amenable for a CC-0 cytoreduction. In addition, the ctDNA levels must be undetectable (whether present or not at baseline) after the completion of induction chemotherapy. ctDNA analysis will be performed using 
Guardant360 CDx and/or Signatera ${ }^{\mathrm{TM}}$. For consistency, we will use Guardant $360 \mathrm{CDx}$ as a selection tool for the trial and Signatera ${ }^{\mathrm{TM}}$ for the exploratory analysis. Informed consents will be obtained from all eligible patients according to the institutional review board requirements.

Patients who progress on induction chemotherapy, have bone and central nervous system metastasis, malignant pleural effusions, histology other than adenocarcinoma and/or previous or current additional malignancies will be excluded (exceptions include history of treated cutaneous basal cell carcinoma, cutaneous squamous cell carcinoma, prostate cancer, or carcinoma in situ). Patients who previously received ablative treatment for metastatic disease (surgery, radiation, or chemotherapy) will be excluded; however, those that have initiated systemic chemotherapy consistent with the trial protocol may participate in the trial if all other eligibility criteria are satisfied. Other exclusion criteria are age $<18$ years or $>80$ years; major surgery within 3 weeks of first trial intervention; $>20 \%$ weight loss despite adequate nutritional interventions; inadequate organ function (Appendix B, Supplementary file); malabsorption or bowel obstruction; active COVID-19 or tubercular infection; and any immunocompromised state including infection with HIV. Pregnant and lactating females will be excluded. The participants must agree to use contraception (hormonal, barrier birth control, or abstinence) for at least 95 days after completing any study interventions.

\section{Randomization}

Once eligibility has been established and patient details have been noted, patients will undergo induction chemotherapy as per the current guidelines. After the completion of chemotherapy, ctDNA levels will be tested, and those having undetectable levels will be allocated in a 1:1 ratio either to the sequential definitive interventions (Arm 1) or to continued chemotherapy (Arm 2). Randomization will be performed using the method of permuted blocks and implemented using a computer-based randomization program. The patients will be stratified by type of primary (intestinal vs pancreatico-biliary); synchronous vs metachronous metastasis; and the response to chemotherapy based on the Response Assessment Criteria in Solid Tumors (RECIST) 1.1 criteria (partial or complete response vs stable disease). Data will be analyzed on an intention to treat (ITT) basis if patients are not subjected to the randomized treatment modality; a modified ITT (mITT) will evaluate outcomes in those patients receiving the intended therapies in each arm. Figure 1 describes the flow schema of the study.

\section{Arm 1: Sequential Cytoreductive Interventions}

Following 3 months of induction chemotherapy and a negative ctDNA assay, patients randomized to the intervention arm will undergo cytoreductive interventions. All sequential interventions must be completed within the 3-month time frame following the randomization. Interventions will generally be performed in the following preferred order: pulmonary lesions first, followed by nonregional nodes, then liver lesions, then primary site, and finally peritoneal disease. However, alteration of this sequence may be allowed after discussion in a multidisciplinary tumor board or at the discretion of the treating physician.

Lung metastases will be addressed either surgically (preferred if feasible) or otherwise via consolidative radiation or ablation. The surgical approach will be either by video-assisted thoracoscopic surgery (VATS) or an open thoracotomy; with a resection maximum of three wedge resections, or a single lobectomy. Either surgical excision or consolidative radiation will address any identified nonregional nodal disease. Excision will be performed for grossly positive nodes as determined clinically or on imaging if undergoing surgery. Similarly, either resection (hepatic wedge resection, sectorectomy, sectionectomy or lobectomy), percutaneous ablation (radiofrequency or microwave), consolidative radiation, or any combination of these treatments will be used for any hepatic lesions depending on location and quantity. The primary site will be managed according to the standard guidelines for the curative-intent treatment, either a surgical resection, radiation, or chemoradiation. Finally, peritoneal metastases will be addressed with a segmental peritonectomy exclusively involving the diseased site; however, no hyperthermic intraperitoneal chemotherapy (HIPEC) will be performed. Of note, the primary site, and any liver and/ or peritoneal lesions may be addressed simultaneously in a single operation if deemed feasible.

Following the completion of sequential cytoreductive therapy, patients will resume standard first-line systemic therapy (including maintenance therapy) until first disease progression, for a duration determined at the treating physician's discretion. At the time of first progression, [if the progression of the disease is determined by imaging to be outside the planned ablative field(s)], patients will proceed to standard second-line systemic therapy. A repeat metastasectomy may be considered for patients who present with metachronous oligo-recurrence (oligometastasis appearing 6 months after the initial cytoreductive interventions) that is amenable for resection/ablation with curative intent. ctDNA levels will be measured after each intervention and monthly thereafter for the first 6 months after completion of interventions (Fig. 1). 


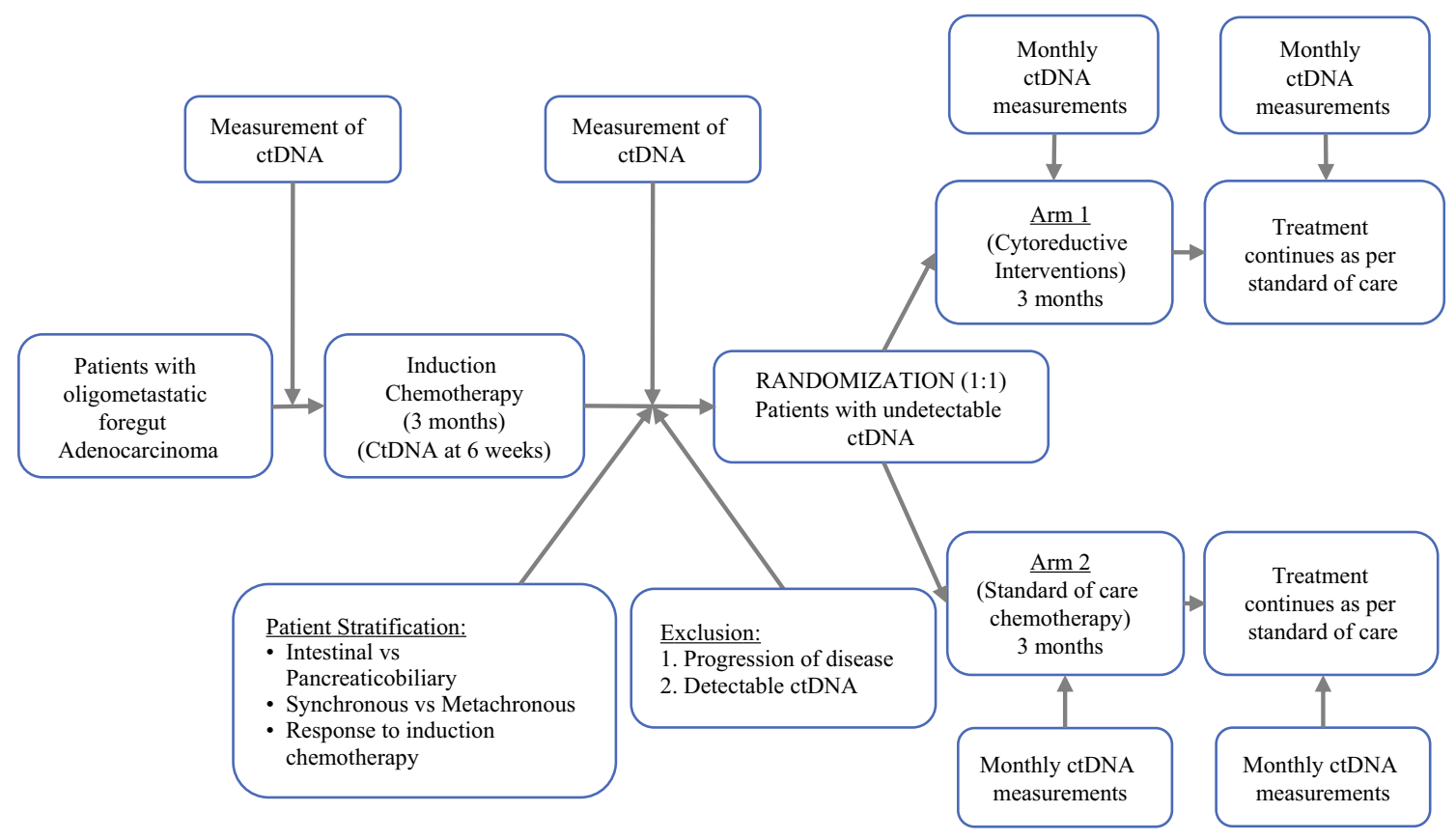

FIG. 1 Trial schema

Arm 2: Chemotherapy

The patients randomized to the continued systemic therapy arm will continue on as per current guidelines until progression. Disease progression will be determined by cross-sectional imaging as outlined in the primary endpoints. ctDNA levels will be measured monthly for 6 months. If first disease progression is confirmed, patients will be eligible for second-line palliative therapies as appropriate. If a patient discontinues therapy for whatever reason without disease progression, patients will then be followed for disease progression or death every 2-3 months until the study completion.

\section{Follow-Up}

Monthly follow-up will be carried out for 12 months (Tables 1, 2). Subsequently, patients will be followed as per current recommendations for the respective primary site. Patients will undergo cross-sectional imaging every 2 months to assess for disease progression. In addition, ctDNA levels will be assessed monthly for the first 6 months and subsequently every 2 months until 2 years after randomization. If a participant fails to return to the clinic for a required study visit, every effort will be made to regain contact, and the missed visit will be rescheduled. These contact attempts will be documented in the participant's medical record. The participant will not be considered lost to follow-up until the last scheduled visit.

\section{Endpoints}

The primary endpoint of this trial is progression-free survival (PFS), from the time from randomization to clinical disease progression or death, whichever comes first. Progression will be determined on cross-sectional imaging based on the RECIST 1.1 criteria. The first imaging for the assessment of progression will be 3 months after randomization in both arms (i.e., after the completion of all planned cytoreductive interventions). Any disease progression noted on imaging intended for therapeutic planning obtained before a cytoreductive intervention within these first 3 months will be designated as a progressive disease only if there is a confirmed out-of-field disease or a progression precluding planned cytoreductive intervention.

Secondary endpoints include the ctDNA conversion rates post-induction therapy, overall survival (OS), diseasefree survival (DFS) in the intervention arm, 6-month PFS rate, 12-month OS rate, 6- and 12-month ctDNA negativity rates, ctDNA PFS, $\mathrm{PFS}_{2}$ (defined as time from randomization to progression on second-line therapy, which includes repeat interventions as indicated in the interventions arm), adverse events defined as per Common Terminology Criteria for Adverse Events (CTCAE) version 5.0, and finally, the patient-reported outcomes (PRO) for quality of life and financial toxicity. The quality of life will be assessed using the EORTC-QLQ-C30 questionnaire, ${ }^{36}$ while financial toxicity will be assessed using the Comprehensive Score for Financial Toxicity (COST) 
Phase II Prospective, Open-Label Randomized Controlled Trial

4587

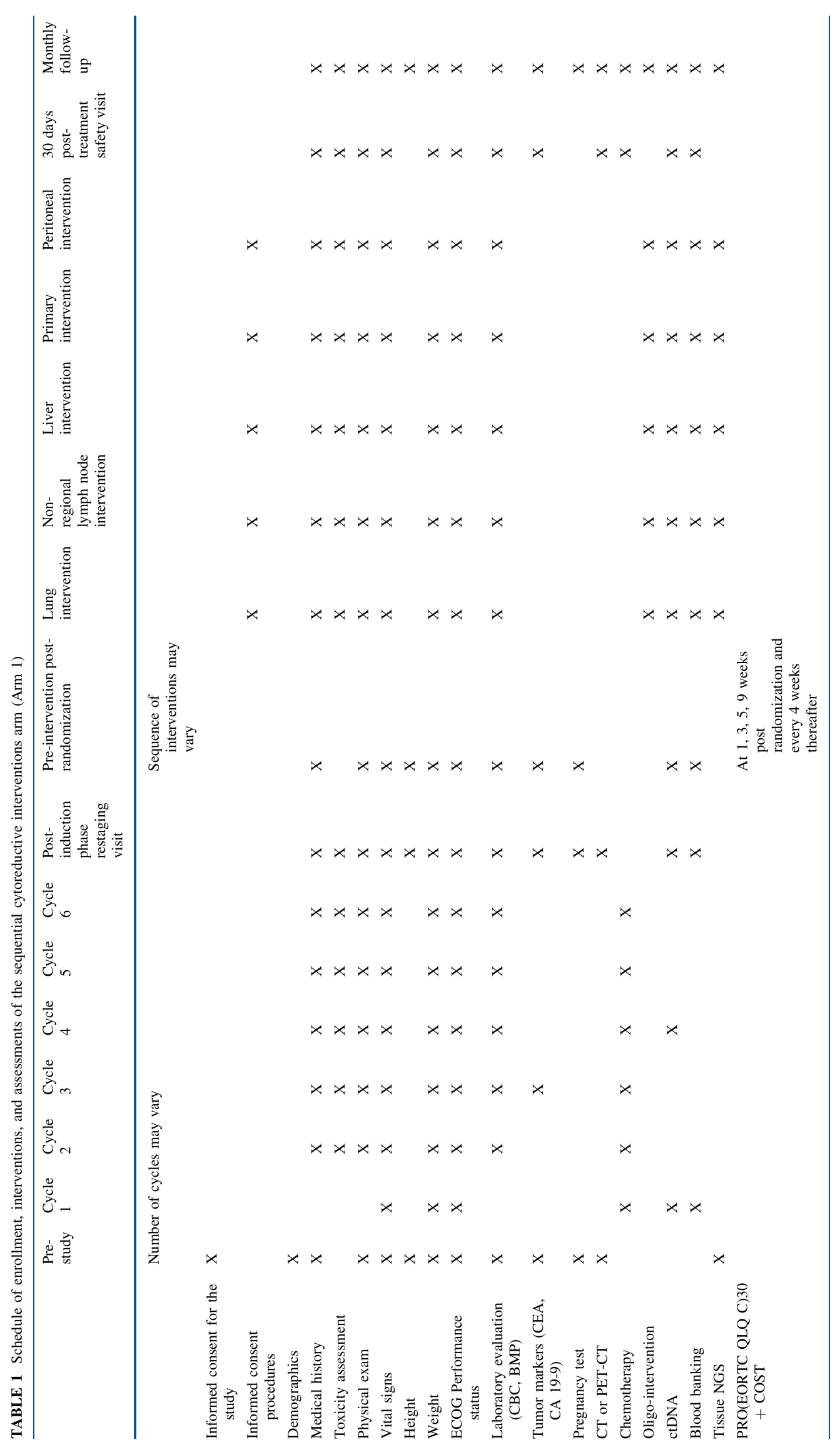


questionnaire. ${ }^{37}$ PROs will be completed after randomization on weeks $1,3,5$, and 9 , and every 4 weeks thereafter, at the treatment-discontinuation visit, and the 30-day posttreatment safety follow-up visit. A subgroup analysis will be conducted for these outcomes in various stratification subgroups. Exploratory endpoints will include molecular characterization of the primary and metastatic disease sites to determine prognostic biomarkers.

\section{Statistical Analysis}

This study is designed to detect an improvement in the median PFS from 3 months in the standard of care arm (Arm 2) to 6 months in the intervention arm (Arm 1), corresponding to a hazard ratio (HR) of 0.5 (under exponential survival assumptions). This is based on the published range of PFS of standard of care therapy for patients with foregut adenocarcinoma from the point of randomization (after 3 months of induction chemotherapy). Patient enrolment will be conducted on a rolling basis. The required sample size for the primary endpoint of PFS based on using a one-sided alpha of 0.1 and power of $80 \%$ is 44 patients ( 22 per arm). We will enroll patients for 24 months and have a subsequent follow-up of 12 months. To account for an estimated $10 \%$ dropout, we will increase the total enrolment to 48 patients. The projected number of PFS events is 42 . If fewer events have occurred at the end of 3 years, follow-up will continue until 42 PFS events have been observed.

The baseline continuous data will be expressed as means, standard deviations, medians, and interquartile ranges; baseline categorical data will be described as numbers and percentages. All comparative analyses will be conducted on an 'intention to treat' basis; a modified intention to treat (mITT) will evaluate outcomes in those patients receiving the intended therapies in each arm. PFS, OS, and ctDNA PFS will be estimated by the Kaplan-Meier method and compared between the two treatment arms using a log-rank test. ${ }^{38}$

A Cox regression model will be fitted to estimate the HR along with a $90 \%$ confidence interval (CI). The Brookmeyer-Crowley procedure will be performed to estimate median event times, along with $90 \%$ CIs. ${ }^{39}$ Mixed-effects regression models will be fitted to analyze longitudinal ctDNA measurements, the quality of life (PRO) outcomes, and to compare the profiles over time between the two treatment arms.

Surgical morbidity and other adverse events will be summarized by type, grade, and attribution, and compared between groups using chi-square or Fisher exact tests, as appropriate. Finally, the correlation among biomarkers will be summarized by Pearson or Spearman rank correlation coefficients, and the association between baseline biomarkers and PFS, OS, and ctDNA PFS will be analyzed via Cox regression modeling. These models will include random subject effects in addition to treatment, time, and treatment-by-time interaction terms.

\section{Accrual and Limitations}

For the inclusion of 48 subjects, approximately 24 months will be required. We will attempt to enroll 24 patients each year for 2 consecutive years. A futility analysis will be performed after half of the projected 42 PFS events have occurred, using the Wieand et al. (1994) stopping criterion. ${ }^{40}$ If, at this point, the $\mathrm{HR}$ for the experimental to control arm is $>1$, i.e., aggressive treatment is performing no better than the standard of care, the trial will be terminated for futility. This rule is associated with a minimal power loss $(<2 \%)$.

\section{Monitoring and Auditing}

This study will be monitored by the designated University of Chicago Clinical Research Associate (CRA) as per the University of Chicago, Section of Hematology and Oncology standard operating procedures. Monitoring will be conducted to verify the following: the adherence to the protocol, completeness and accuracy of study data and samples collected, compliance with regulations, and submission of required source documents.

Quality assurance audits will ensure that study data are collected, documented, and reported in compliance with Good Clinical Practices (GCP) Guidelines and regulatory requirements. The audit will review subjects enrolled at the University of Chicago as per audit procedures specified in the University of Chicago Comprehensive Cancer Center (UC CCC) Data and Safety Monitoring Plan.

\section{Trial Registration}

The trial is registered at http://clinicaltrials.g ov (NCT04931420).

\section{DISCUSSION}

Cancers of foregut origin pose a considerable burden on society with more than 160,000 estimated new cases in 2021 , leading to more than 100,000 estimated deaths in the United States. ${ }^{41}$ Forty percent of esophageal cancer, 36\% of gastric cancer, and more than $50 \%$ of pancreatic cancer patients have metastatic disease at the time of diagnosis. The majority succumb to their disease process within 18 months of diagnosis. Therefore, the National Comprehensive Cancer Network (NCCN) guidelines recommend enrolling such patients in clinical trials. ${ }^{2-4}$ Our study is the 
first of its kind and will attempt to identify a subset of patients with metastatic foregut adenocarcinoma who may benefit from sequential cytoreductive interventions in addition to the standard of care therapy.

The concept of oligometastasis was first described by Hellman and Weichselbaum in 1995 as a characteristic of various solid tumors having limited metastatic capacity. ${ }^{14}$ This limited metastatic capacity relates not only to the small number of lesions confined to limited sites, but also potentially to the favorable tumor biology, which may provide clinicians with a window of opportunity in which to intervene and to delay or prevent malignant progression. ${ }^{15,42}$ On the other hand, oligometasastic presentation could merely represent lead-time bias, with numerous occult metastatic lesions not yet visible - this is particularly true historically for the upper GI cancers. However, over the past decade, a few studies involving patients with various solid malignancies have strengthened this concept of heterogeneity in metastatic potential. In a multicenter randomized study, Gomez et al. showed better PFS in patients with NSCLC having three or fewer metastatic lesions treated with localized consolidative radiation therapy in addition to the standard maintenance therapy. ${ }^{17}$ Reducing the tumor burden with locoregional therapies such as surgery, radiation, or ablation has improved survival for some histologies such as colon cancer. ${ }^{43-45}$ The recent SABR-COMET trial found that multi-site radiation for oligometastatic disease improved survival in the cohort containing numerous solid tumors. ${ }^{45}$ However, there is a scarcity of randomized data for the foregut tumors showing the benefit of aggressive interventions in the setting of metastatic or oligometastatic disease. The AIO-FLOT3 trial demonstrated improvement in overall survival in patients having limited metastatic gastric or gastroesophageal junction adenocarcinoma who underwent complete resection of the primary tumor and had complete macroscopic response at metastatic sites after neoadjuvant chemotherapy. ${ }^{13}$ The REGATTA trial, however, failed to show a benefit to gastrectomy (but not metastasectomy) over standard chemotherapy in patients with single-site metastasis from gastric cancer. ${ }^{46}$ However, not all sites of disease in this study were definitively addressed with surgery and/or ablation. A handful of retrospective cohort studies have demonstrated favorable oncological outcomes with curative-intent treatment for oligometastatic cancers of the stomach, esophagogastric junction, pancreas, and bile duct. $^{6,9,10,16}$ The major limitations of these studies are their limited sample size, heterogeneity in definitions of oligometastasis and treatment protocols, and lack of randomized control to rule out latent biology and selection bias. Importantly, with increasing efficacy of systemic therapies for upper GI cancers including more potent triplet (and even quadruplet) cytotoxic chemotherapy regimens as well as immunotherapy checkpoint inhibitors, there is possibly more potential that surgical/ablative approaches may provide benefit in oligometastatic scenarios. However, it remains crucial to sensibly identify which patients will actually derive meaningful benefit.

Currently, liquid biopsies, such as assays for ctDNA along with ctDNA dynamics before and after systemic therapy, provide for a non-invasive, rapid assessment tool of prognosis. Studies have established the role of ctDNA in prognostication after curative-intent surgery for colon cancers and some foregut cancers such as gastric cancer, ${ }^{23,47}$ and it has been described as a prognostic marker, and used for postoperative surveillance in both diseases. ${ }^{22,23,48}$ Pietrasz et al. demonstrated that the presence of ctDNA was strongly correlated with poor overall survival in patients with advanced pancreatic or ampullary adenocarcinoma. ${ }^{24} \mathrm{~A}$ subgroup analysis demonstrated that patients with undetectable ctDNA following surgery had a superior diseasefree survival (17.9 vs 4.6 months) and overall survival (32.2 vs 19.3 months). ${ }^{24}$ Several groups, including ours, have demonstrated that in the metastatic setting, ctDNA dynamics before and after therapy have prognostic implications, with patients having stable or increasing ctDNA levels having worse prognoses than those with a $50 \%$ or more decline in ctDNA levels. Thus, a major hypothesis of the current study will evaluate the utility of ctDNA as a biomarker to identify favorable tumor biology to judiciously select patients most suitable to proceed with aggressive therapies in the oligometastatic setting.

This trial protocol has various potential limitations. The trial involves enrolment of various histologies, which might confound the results due to the differing clinical and biological behavior. We have tried to address this by stratifying the primary sites based on whether they are of intestinal or pancreaticobiliary origin, prior to randomization. Another limitation is the multifactorial selection criteria, particularly requiring undetectable ctDNA at enrolment, which may lead to a low accrual rate. However, given the poor prognosis of this collection of cancers, we have opted to aggressively select patients for good prognosis, then evaluate this population in randomized fashion in order to determine if there is benefit to aggressive intervention versus continued palliative systemic therapy. We are also limiting cytoreductive interventions to be completed within 3 months, which may not allow all the interventions to be performed in a subset of cases.

The study has potential strengths. If successful, this study will advance the field by providing evidence for or against the provision of invasive treatment options of common and aggressive foregut tumors for patients with limited metastatic disease that is ctDNA negative after induction therapy. The study may also yield insights into the molecular characteristics of patients with superior 
4590

A. Dhiman et al.

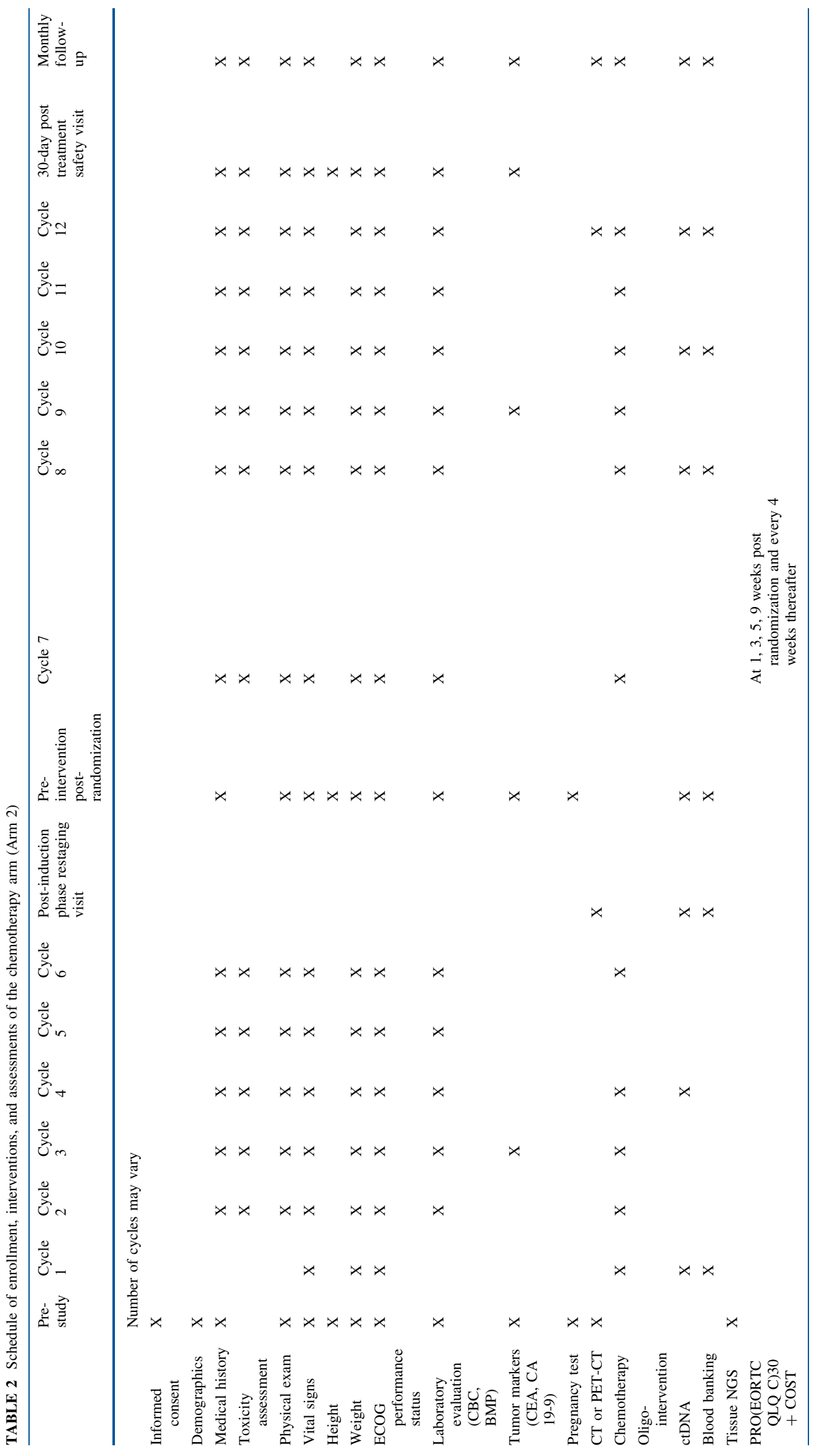


outcomes, leading to better selection criteria for future research. This study design also allows for adequate monitoring of the feasibility and safety of these aggressive interventions in terms of adverse events, patient quality of life, and financial burden.

This randomized clinical trial aims to provide the evidence for effectiveness and safety of curative-intent interventions in patients with ctDNA negative oligometastatic adenocarcinoma of foregut origin and to identify the subset of patients who may benefit most from these interventions. Moreover, data from this study will likely inform the design of a larger, phase III trial and develop pertinent, timely, and relevant knowledge in the field of oncology.

Supplementary Information The online version contains supplementary material available at https://doi.org/10.1245/s10434021-11249-7.

DISCLOSURES Daniel V.T. Catenacci, MD, reports personal fees for Genentech/Roche, Eli Lilly, Merck, Daiichi Sankyo, BMS, Ono, Five Prime, Seattle Genetics, Amgen, Taiho, Astellas, Gritstone, Pieris, Zymeworks, Basilea, QED, Arcus, Foundation Medicine, Pierian, Silverback Therapeutics, Servier, Blueprint Medicines, Arcus Biosciences, Tempus, Guardant Health, Archer, and Natera. Osmanuddin Ahmed reports Advisory boards: Johnson and Johnson, Boston Scientific, Medtronic; Speaking fees: Agron Medical, Canon Medical, Philips, Penumbra; Research Grant: Canon Medical. Sean Pitroda: Two patents for methods and kits for diagnosis and triage of patients with colorectal liver metastases are pending. Andy Liao received consultation honoraria for Ispen, Exelixis, Eisai, Genentech, QED Therapeutics, Incyte, Lexicon, BluePrints Medicine, Transthera, Histosonics. Ralph Weichselbaum has stock and other ownership interests with Boost Therapeutics, Immvira LLC, Reflexion Pharmaceuticals, Coordination Pharmaceuticals Inc., Magi Therapeutics, Oncosenescence. He has served in a consulting or advisory role for Aettis Inc., Astrazeneca, Coordination Pharmaceuticals, Genus, Merck Serono S.A., Nano proteagen, NKMax America Inc, Shuttle Pharmaceuticals, Highlight Therapeutics, S.L. He has research grants with Varian and Regeneron. He has received compensation including travel, accommodations, or expense reimbursement from Astrazeneca, Boehringer Ingelheim Ltd. and Merck Serono S.A. All other authors have no disclosures to declare.

\section{REFERENCES}

1. Sung H, Ferlay J, Siegel RL, et al. Global cancer statistics 2020: GLOBOCAN estimates of incidence and mortality worldwide for 36 cancers in 185 countries. CA Cancer J Clin. 2021;71(3):209-49. https://doi.org/10.3322/caac.21660.

2. Ajani JA, D'Amico TA, Almhanna K, et al. Gastric cancer, version 3.2016, NCCN clinical practice guidelines in oncology. $J$ Natl Compr Canc Netw. 2016;14(10):1286-312. https://doi.org/ 10.6004/jnccn.2016.0137.

3. Ajani JA, D'Amico TA, Bentrem DJ, et al. Esophageal and esophagogastric junction cancers, version 2.2019, NCCN clinical practice guidelines in oncology. J Natl Compr Canc Netw. 2019;17(7):855-83. https://doi.org/10.6004/jnccn.2019.0033.

4. Tempero MA, Malafa MP, Chiorean EG, et al. Pancreatic adenocarcinoma, version 1.2019. J Natl Compr Canc Netw. 2019;17(3):202-10. https://doi.org/10.6004/jnccn.2019.0014.
5. Al-Batran SE, Hofheinz RD, Reichart A, et al. Quality of life and outcome of patients with metastatic pancreatic cancer receiving first-line chemotherapy with nab-paclitaxel and gemcitabine: real-life results from the prospective QOLIXANE trial of the platform for outcome, quality of life and translational research on pancreatic cancer registry. Int J Cancer. 2021;148(6):1478-88. h ttps://doi.org/10.1002/ijc.33336.

6. Frigerio I, Regi P, Giardino A, et al. Downstaging in stage IV pancreatic cancer: a new population eligible for surgery? Ann Surg Oncol. 2017;24(8):2397-403. https://doi.org/10.1245/s104 34-017-5885-4.

7. Han DS, Suh YS, Kong SH, et al. Outcomes of surgery aiming at curative resection in good responder to induction chemotherapy for gastric cancer with distant metastases. J Surg Oncol. 2013;107(5):511-6. https://doi.org/10.1002/jso.23284.

8. Kandel P, Wallace MB, Stauffer J, et al. Survival of patients with oligometastatic pancreatic ductal adenocarcinoma treated with combined modality treatment including surgical resection: a pilot study. J Pancreat Cancer. 2018;4(1):88-94. https://doi.org/10. 1089/pancan.2018.0011.

9. Schmidt T, Alldinger I, Blank S, et al. Surgery in oesophagogastric cancer with metastatic disease: treatment, prognosis and preoperative patient selection. Eur $J$ Surg Oncol. 2015;41(10):1340-7. https://doi.org/10.1016/j.ejso.2015.05.005.

10. Shrikhande SV, Kleeff J, Reiser C, et al. Pancreatic resection for M1 pancreatic ductal adenocarcinoma. Ann Surg Oncol. 2007;14(1):118-27. https://doi.org/10.1245/s10434-006-9131-8.

11. Tachezy M, Gebauer F, Janot M, et al. Synchronous resections of hepatic oligometastatic pancreatic cancer: disputing a principle in a time of safe pancreatic operations in a retrospective multicenter analysis. Surgery. 2016;160(1):136-44. https://doi.org/10.1016/j. surg.2016.02.019.

12. Wright GP, Poruk KE, Zenati MS, et al. Primary tumor resection following favorable response to systemic chemotherapy in stage IV pancreatic adenocarcinoma with synchronous metastases: a biinstitutional analysis. J Gastrointest Surg. 2016;20(11):1830-5. h ttps://doi.org/10.1007/s11605-016-3256-2.

13. Al-Batran SE, Homann N, Pauligk C, et al. Effect of neoadjuvant chemotherapy followed by surgical resection on survival in patients with limited metastatic gastric or gastroesophageal junction cancer: the AIO-FLOT3 trial. JAMA Oncol. 2017;3(9):1237-44. https://doi.org/10.1001/jamaoncol.2017. 0515.

14. Hellman S, Weichselbaum RR. Oligometastases. J Clin Oncol. 1995;13(1):8-10. https://doi.org/10.1200/jco.1995.13.1.8.

15. Weichselbaum RR, Hellman S. Oligometastases revisited. Nat Rev Clin Oncol. 2011;8(6):378-82. https://doi.org/10.1038/nrcli nonc.2011.44.

16. Franzese $\mathrm{C}$, Bonu ML, Comito $\mathrm{T}$, et al. Stereotactic body radiotherapy in the management of oligometastatic and recurrent biliary tract cancer: single-institution analysis of outcome and toxicity. J Cancer Res Clin Oncol. 2020;146(9):2289-97. http s://doi.org/10.1007/s00432-020-03285-9.

17. Gomez DR, Blumenschein GR Jr, Lee JJ, et al. Local consolidative therapy versus maintenance therapy or observation for patients with oligometastatic non-small-cell lung cancer without progression after first-line systemic therapy: a multicentre, randomised, controlled, phase 2 study. Lancet Oncol. 2016;17(12):1672-82. https://doi.org/10.1016/s1470-2045(16)30 $532-0$.

18. Ruers T, Punt C, Van Coevorden F, et al. Radiofrequency ablation combined with systemic treatment versus systemic treatment alone in patients with non-resectable colorectal liver metastases: a randomized EORTC Intergroup phase II study (EORTC 40004). Ann Oncol. 2012;23(10):2619-26. https://doi.org/10.1093/annon c/mds053. 
19. Ruers T, Van Coevorden F, Punt CJ, et al. Local treatment of unresectable colorectal liver metastases: results of a randomized phase II trial. J Natl Cancer Inst. 2017. https://doi.org/10.1093/ jnci/djx015.

20. Ost P, Reynders D, Decaestecker K, et al. Surveillance or metastasis-directed therapy for oligometastatic prostate cancer recurrence: a prospective, randomized, multicenter phase II trial. J Clin Oncol. 2018;36(5):446-53. https://doi.org/10.1200/jco.20 17.75.4853.

21. Ma M, Zhu H, Zhang C, Sun X, Gao X, Chen G. "Liquid biopsy"-ctDNA detection with great potential and challenges. Ann Transl Med. 2015;3(16):235. https://doi.org/10.3978/j.issn. 2305-5839.2015.09.29.

22. Spindler KL, Appelt AL, Pallisgaard N, Andersen RF, Brandslund I, Jakobsen A. Cell-free DNA in healthy individuals, noncancerous disease and strong prognostic value in colorectal cancer. Int J Cancer. 2014;135(12):2984-91. https://doi.org/10. 1002/ijc.28946.

23. Maron SB, Chase LM, Lomnicki S, et al. Circulating tumor DNA sequencing analysis of gastroesophageal adenocarcinoma. Clin Cancer Res. 2019;25(23):7098-112. https://doi.org/10.1158/107 8-0432.Ccr-19-1704.

24. Pietrasz D, Pécuchet N, Garlan F, et al. Plasma circulating tumor DNA in pancreatic cancer patients is a prognostic marker. Clin Cancer Res. 2017;23(1):116-23. https://doi.org/10.1158/1078-0 432.Ccr-16-0806.

25. Parikh AR, Van Seventer EE, Siravegna G, et al. Minimal residual disease detection using a plasma-only circulating tumor DNA assay in colorectal cancer patients. Clin Cancer Res. 2021. https://doi.org/10.1158/1078-0432.ccr-21-0410.

26. Odegaard JI, Vincent JJ, Mortimer S, et al. Validation of a plasma-based comprehensive cancer genotyping assay utilizing orthogonal tissue- and plasma-based methodologies. Clin Cancer Res. 2018;24(15):3539-49. https://doi.org/10.1158/1078-0432.Cc r-17-3831.

27. Bettegowda C, Sausen M, Leary RJ, et al. Detection of circulating tumor DNA in early- and late-stage human malignancies. Sci Transl Med. 2014;6(224):224ra24. https://doi.org/10.1126/sc itranslmed.3007094.

28. Gao Y, Xi H, Wei B, et al. Association between liquid biopsy and prognosis of gastric cancer patients: a systematic review and meta-analysis. Front Oncol. 2019;9:1222. https://doi.org/10.338 9/fonc.2019.01222.

29. Tie J, Cohen JD, Wang Y, et al. Circulating tumor DNA analyses as markers of recurrence risk and benefit of adjuvant therapy for stage III colon cancer. JAMA Oncol. 2019;5(12):1710-7. https://d oi.org/10.1001/jamaoncol.2019.3616.

30. Rumiato E, Boldrin E, Malacrida S, et al. Detection of genetic alterations in cfDNA as a possible strategy to monitor the neoplastic progression of Barrett's esophagus. Transl Res. 2017;190:16-24.e1. https://doi.org/10.1016/j.trsl.2017.09.004.

31. Hu ZY, Xie N, Tian C, et al. Identifying circulating tumor DNA mutation profiles in metastatic breast cancer patients with multiline resistance. EBioMedicine. 2018;32:111-8. https://doi.org/ 10.1016/j.ebiom.2018.05.015.

32. Tie J, Wang $\mathrm{Y}$, Tomasetti C, et al. Circulating tumor DNA analysis detects minimal residual disease and predicts recurrence in patients with stage II colon cancer. Sci Transl Med. 2016;8(346):346ra92. https://doi.org/10.1126/scitranslmed.aaf 6219.

33. Garcia-Murillas I, Schiavon G, Weigelt B, et al. Mutation tracking in circulating tumor DNA predicts relapse in early breast cancer. Sci Transl Med. 2015;7(302):302ra133. https://doi.org/10. 1126/scitranslmed.aab0021.

34. Chaudhuri AA, Chabon JJ, Lovejoy AF, et al. Early detection of molecular residual disease in localized lung cancer by circulating tumor DNA profiling. Cancer Discov. 2017;7(12):1394-403. h ttps://doi.org/10.1158/2159-8290.Cd-17-0716.

35. Chan AW, Tetzlaff JM, Altman DG, et al. SPIRIT 2013 statement: defining standard protocol items for clinical trials. Ann Intern Med. 2013;158(3):200-7. https://doi.org/10.7326/0003-48 19-158-3-201302050-00583.

36. Aaronson NK, Ahmedzai S, Bergman B, et al. The European organization for research and treatment of cancer QLQ-C30: a quality-of-life instrument for use in international clinical trials in oncology. J Natl Cancer Inst. 1993;85(5):365-76. https://doi.org/ 10.1093/jnci/85.5.365.

37. de Souza JA, Yap BJ, Hlubocky FJ, et al. The development of a financial toxicity patient-reported outcome in cancer: The COST measure. Cancer. 2014;120(20):3245-53. https://doi.org/10.100 2/cncr.28814.

38. Kaplan EL, Meier P. Nonparametric estimation from incomplete observations. J Am Stat Assoc. 1958;53(282):457. https://doi.org/ $10.2307 / 2281868$

39. Brookmeyer R, Crowley J. A confidence interval for the median survival time. Biometrics. 1982;38(1):29. https://doi.org/10.2307/ 2530286.

40. Wieand S, Schroeder G, O'Fallon JR. Stopping when the experimental regimen does not appear to help. Stat Med. 1994;13(13-14):1453-8. https://doi.org/10.1002/sim. 4780131321.

41. Siegel RL, Miller KD, Fuchs HE, Jemal A. Cancer statistics, 2021. CA Cancer J Clin. 2021;71(1):7-33. https://doi.org/10.33 22/caac. 21654.

42. Pitroda SP, Weichselbaum RR. Integrated molecular and clinical staging defines the spectrum of metastatic cancer. Nat Rev Clin Oncol. 2019;16(9):581-8. https://doi.org/10.1038/s41571-019-02 20-6.

43. Kemeny NE, Chou JF, Boucher TM, et al. Updated long-term survival for patients with metastatic colorectal cancer treated with liver resection followed by hepatic arterial infusion and systemic chemotherapy. J Surg Oncol. 2016;113(5):477-84. https://doi.or $\mathrm{g} / 10.1002 / \mathrm{j}$ so. 24189 .

44. Okumura T, Boku N, Hishida T, et al. Surgical outcome and prognostic stratification for pulmonary metastasis from colorectal cancer. Ann Thorac Surg. 2017;104(3):979-87. https://doi.org/10. 1016/j.athoracsur.2017.03.021.

45. Palma DA, Olson R, Harrow S, et al. Stereotactic ablative radiotherapy versus standard of care palliative treatment in patients with oligometastatic cancers (SABR-COMET): a randomised, phase 2, open-label trial. Lancet. 2019;393(10185):2051-8. https://doi.org/10.1016/s0140-6736(18 $32487-5$.

46. Fujitani K, Yang HK, Mizusawa J, et al. Gastrectomy plus chemotherapy versus chemotherapy alone for advanced gastric cancer with a single non-curable factor (REGATTA): a phase 3, randomised controlled trial. Lancet Oncol. 2016;17(3):309-18. h ttps://doi.org/10.1016/s1470-2045(15)00553-7.

47. Leal A, Van Grieken NCT, Palsgrove DN, et al. White blood cell and cell-free DNA analyses for detection of residual disease in gastric cancer. Nat Commun. 2020. https://doi.org/10.1038/s414 67-020-14310-3.

48. Spindler KL, Pallisgaard N, Vogelius I, Jakobsen A. Quantitative cell-free DNA, KRAS, and BRAF mutations in plasma from patients with metastatic colorectal cancer during treatment with cetuximab and irinotecan. Clin Cancer Res. 2012;18(4):1177-85. https://doi.org/10.1158/1078-0432.Ccr-11-0564.

Publisher's Note Springer Nature remains neutral with regard to jurisdictional claims in published maps and institutional affiliations. 